\title{
Riqueza de mamíferos del Parque Nacional Barranca del Cupatitzio, Michoacán, México
}

\author{
Mammal richness from Barranca del Cupatitzio National Park, Michoacán, Mexico
}

\author{
Gilberto Chávez-León ${ }^{1 *}$ y Sergio Zaragoza Rivera² \\ Instituto Nacional de Investigaciones Forestales, Agrícolas y Pecuarias, Av. Latinoamericana 1101, 60150 Uruapan, Michoacán, México. \\ ${ }^{2}$ Galaxias 303, Fracc. Cosmos, 58011, Morelia, Michoacán, México. \\ * Correspondencia: chavez.josegilberto@inifap.gob.mx
}

\begin{abstract}
Resumen. En este trabajo se documenta la riqueza de mamíferos del Parque Nacional Barranca del Cupatitzio, Michoacán, México, registrada de abril de 2003 a octubre de 2004 mediante diversos métodos de campo. Se identificaron 43 especies, pertenecientes a 8 órdenes, 16 familias y 32 géneros. El estimador no paramétrico ACE indicó que podrían registrarse al menos 13 especies más y que se logró detectar el $77 \%$ de las que posiblemente existen en el parque. Los mamíferos voladores constituyeron el $25.6 \%$ del total de las especies y los no voladores el $74.4 \%$. Hay 7 especies (16.3\%) endémicas de México, 5 (11.6\%) endémicas de Mesoamérica, 10 (23.3\%) compartidas con Norteamérica, 7 (16.3\%) con Sudamérica, $13(30.2 \%)$ con Norte y Sudamérica, y 1 es exótica (2.3\%). Por sus afinidades biogeográficas, 22 especies (52.4\%) son neárticas, 16 (38.1\%) neotropicales y 4 (9.5\%) transicionales. (4.7\%) La lista de la Norma Oficial Mexicana 059 incluye 2 especies:, 1 en peligro de extinción (Leopardus wiedii) y 1 amenazada (Crateogomys fumosus). Se identificaron 8 especies prioritarias para su conservación considerando criterios de riesgo, endemismo y restricción de distribución. No obstante su poca superficie y ubicación en una zona de crecimiento urbano, Barranca del Cupatitzio es un área de importancia para la conservación de la diversidad de mamíferos, ya que protege poco más de la cuarta parte de las especies de Michoacán.
\end{abstract}

Palabras clave: distribución, endemismo, estado de conservación, especies prioritarias.

\begin{abstract}
In this paper we present field information on mammal richness from Barranca del Cupatitzio National Park, Michoacán, Mexico, recorded from April 2003 to October 2004, by means of specimen collection, visual detection, photo trapping and search of remains, signs and tracks. The area is covered mainly by closed canopy conifer forests. The mammal richness of this park was 43 species belonging to 8 orders, 16 families and 31 genera. The non-parametric ACE estimator indicated that $77 \%$ of all possible species were recorded and at least 13 more species can be potentially found. From all species recorded, $25.6 \%$ were flying mammals and $74.4 \%$ were non-flying. The distribution of species shows that 7 mammals (16.3\%) are endemic to Mexico, 5 (11.6\%) are endemic to Meso America, 10 (23.3\%) are shared with North America, 7 (16.3\%) with South America, 13 (30.2\%) with North and South America, and 1 is exotic (2.3\%). Considering their biogeographic affinities, 22 species $(52.4 \%)$ are Neartic, $16(38.1 \%)$ are Neotropical and $4(9.5 \%)$ are transitional. Two species (4.7\%) are considered at risk by Mexican regulations: 1 in danger of extinction (Leopardus wiedii) and 1 as threatened (Crateogomys fumosus). Applying risk, endemism and distribution criteria, we identified 8 mammals as priority species for conservation in the park. This national park protects more than a quarter of the mammal species from Michoacán and is a critical area for their conservation, nevertheless its small surface and urban encroachment.
\end{abstract}

Key words: distribution, endemism, conservation status, priority species.

\section{Introducción}

El eje volcánico transmexicano es considerado como una de las regiones más diversas en mamíferos y con mayor concentración de endemismos en México ( $\mathrm{Fa}$ y Morales, 1993). En esa zona se encuentra la mayoría de los parques nacionales decretados durante las décadas de 1930 y 1940 con la finalidad de proteger las cuencas

Recibido: 29 mayo 2007; aceptado: 07 noviembre 2008 hidrológicas de las principales ciudades y montañas que ahí se encuentran (Vargas, 1997) y es donde se concentra una de las mayores densidades de población humana del país (INEGI, 2006). A fin de manejar efectivamente estas áreas naturales protegidas es necesario contar con inventarios específicos actualizados que indiquen con que taxones se cuenta y donde se distribuyen, además de que contribuyan al conocimiento de la historia natural de las especies registradas (Janzen, 1997).

Entre esos antiguos parques nacionales se halla 
Barranca del Cupatitzio, caracterizado por encontrarse parcialmente dentro de la ciudad de Uruapan en el estado de Michoacán. No obstante esta cercanía con una zona urbana, su mastofauna es poco conocida. Estudios previos, efectuados entre 1981 y 1985 , registran 62 especies para la sierra purépecha (Orduña y Salas, 1993), que forma parte del eje neovolcánico, así como 32 especies para el parque (Orduña et al., 1993), lo que representa el 38.7 y el 20.0\%, respectivamente, de las 160 especies de mamíferos que se han registrado en Michoacán (Núñez, 2003, 2005).

Ante la necesidad de sustentar el Programa de Conservación y Manejo (PCyM) de esta área natural protegida con información reciente, se actualizaron y verificaron los inventarios biológicos, incluyendo el de mamíferos. La lista de especies generada en este estudio, que es de un total de 43 especies, fue incorporada al PCyM.

El objetivo general de este trabajo fue contribuir a un mejor conocimiento de la mastofauna del Parque Nacional Barranca del Cupatitzio y, específicamente, generar una lista actualizada de especies para incorporarla al PCyM, determinar la riqueza y estado de conservación de los mamíferos, e identificar las especies prioritarias para su conservación.

\section{Materiales y métodos}

El Parque Nacional Barranca del Cupatitzio se localiza en la provincia fisiográfica del eje neovolcánico transmexicano y en la subprovincia neovolcánica tarasca, al centro-occidente del estado de Michoacán (19 $25^{\circ} 11^{\prime \prime}$ - 19²6' 24" N y $102^{\circ} 07^{\prime}$ '40” - 102 04' 20” O, Fig. 1). Se ubica en el límite de los municipios de Uruapan y Nuevo Parangaricutiro, con una extensión de 458.21 hectáreas. El parque está conformado por 2 polígonos con una separación de $1 \mathrm{~km}$ entre sí. El polígono más extenso, conocido como área de montaña tiene una superficie de 438.55 ha, se caracteriza por una topografía heterogénea constituida por un cono y un domo volcánicos de pendiente suave, cañadas de pendiente fuerte, zonas planas y derrames de lava (Gómez-Tagle, 1985). El polígono más pequeño, llamado área de río, con 19.66 ha, está rodeado por la zona urbana de Uruapan, y su topografía es de barranca y algunas zonas planas. La parte más baja del parque (1 640 - $1710 \mathrm{~m} \mathrm{snm}$ ) es donde se encuentra la barranca donde nace el río Cupatitzio; los puntos más altos son el cerro Chiquito (2 $136 \mathrm{~m} \mathrm{snm)} \mathrm{y} \mathrm{la} \mathrm{loma} \mathrm{Larga} \mathrm{(2} 057 \mathrm{~m} \mathrm{snm),}$ ambos en el área de montaña.

De acuerdo con el sistema de clasificación de tipos de clima de Köppen, modificado por García (1981), el área de estudio se encuentra en la zona que corresponde al clima

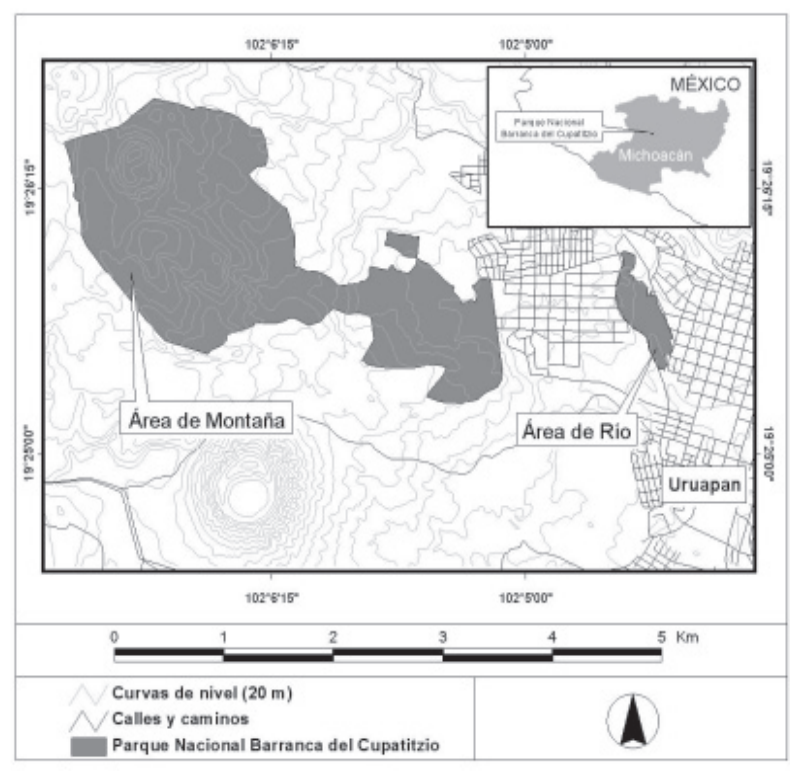

Figura 1. Localización del Parque Nacional Barranca del Cupatitzio, Michoacán, México.

templado semicálido húmedo con abundantes lluvias en verano. Los datos registrados en el periodo 1978 a 2005 en la estación climatológica del parque $\left(19^{\circ} 25^{\prime} 46.5^{\prime \prime} \mathrm{N} \mathrm{y}\right.$ $102^{\circ} 05^{\prime} 34.3^{\prime \prime} \mathrm{O}, 1756 \mathrm{~m} \mathrm{snm}$ ) indican una temperatura media anual de $16.6^{\circ} \mathrm{C}$, con una máxima media anual de $23.9^{\circ} \mathrm{C}$ y mínima media anual de $9.3{ }^{\circ} \mathrm{C}$; la precipitación media anual fue de $1537.4 \mathrm{~mm}$.

La vegetación predominante en el Parque Nacional Barranca del Cupatitzio corresponde al bosque de coníferas con dosel cerrado (Rzedowski, 1978), específicamente bosque de pino-encino y bosque de pino, así como relictos de bosque mesófilo de montaña (Bello y Madrigal, 1996). Las especies arbóreas dominantes, con alturas hasta de 30 m, son Pinus michoacana, P. douglasiana y P. lawsonii, así como Quercus obtusata, Q. castanea y Q. magnoliifolia (Bello y Madrigal, 1996). Las especies asociadas que forman un estrato arbóreo inferior con alturas de 10 a 20 m son Alnus jorullensis, Arbutus xalapensis, Clethra mexicana, Prunus serotina y Ternstroemia pringlei. En el estrato arbustivo, con alturas de 2 a $5 \mathrm{~m}$, se encuentran Ceanothus coeruleus, Lobelia laxiflora, Lupinus bilineatus, Salvia mexicana, Senecio angulifolius y Solanum lanceolatum. El estrato herbáceo está constituido por Adiantum andicola, Asclepias glaucescens, A. otarioides, Begonia gracilis, Dryamaria villosa, Lopezia racemosa, Muhlenbergia ciliata, M. diversiglumis, Pereilema crinitum, Phaseolus coccineus, Piqueria trinervia, Plantago australis, Melinis repens y Sigesbeckia jorullensis.

Los elementos relictuales de bosque mesófilo de 
montaña se distribuyen en barrancas, donde el estrato arbóreo presenta una altura promedio de $15 \mathrm{~m}$, aunque hay pinos dispersos que alcanzan hasta $30 \mathrm{~m}$. Entre las plantas más comunes que lo integran se encuentran: Alnus jorullensis, Bocconia arborea, Carpinus caroliniana, Clethra mexicana, Fraxinus uhdei, Ilex tolucana, Oreopanax salvinii, Prunus serotina y Ternstroemia pringlei (Bello y Madrigal, 1996).

En los 2 polígonos del Parque Nacional se establecieron estaciones permanentes de muestreo, 92 en el área de montaña y 8 en el área de río. Por medio de un sistema de información geográfica (Idrisi32, Clark Labs.) se generó una retícula con inicio aleatorio y una separación de 200 $\mathrm{m}$, formando 100 intersecciones, sobre una ortofoto digital (escala 1:75 000, píxel de 2x2 m). Estas intersecciones con sus respectivas coordenadas geográficas representan los sitios de muestreo, los cuales fueron ubicados en el campo con receptores GPS (Garmin® 12XL y eTrex Legend) y marcados para su posterior relocalización. El trabajo de campo se realizó durante un periodo de 15 meses (abril de 2003 a octubre de 2004). En los sitios se efectuaron muestreos durante 2 o 3 días continuos, cada 2 semanas, mediante colecta de ejemplares, registros visuales $\mathrm{y}$ fotográficos. Los muestreos se complementaron con evidencia indirecta, como huellas y señales (madrigueras, nidos, residuos de comida, senderos y excretas). Las impresiones de huellas se preservaron con yeso dental (Aranda, 2000; Zaragoza, 2006).

La recolecta de mamíferos pequeños se efectuó mediante métodos convencionales (Romero-Almaraz et al., 2000). Para roedores se usaron 150 trampas Sherman ${ }^{\circledR}$ plegables de $30.5 \times 8 \times 9.5 \mathrm{~cm}$, cebadas con una mezcla de hojuelas de avena, esencia de vainilla, crema de cacahuate y plátano en 30 sitios de muestreo. Para colectar tuzas se usaron 10 trampas Volke ${ }^{\circledR}$ colocadas dentro de sus galerías en 15 sitios de muestreo. El método de trampeo consistió en transectos y cuadrantes, colocando las trampas al atardecer separadas una de la otra cada 10 $\mathrm{m}$, revisándolas al amanecer (Romero-Almaraz et al., 2000). Los murciélagos fueron colectados en 25 sitios de muestreo con redes ornitológicas de $12 \mathrm{~m}$ de longitud, $2.6 \mathrm{~m}$ de alto, malla de $30 \mathrm{~mm}$ y 4 paneles. En los sitios de muestreo se mantuvieron abiertas las redes desde el atardecer hasta el amanecer revisándolas constantemente. A los ejemplares se les tomaron medidas somáticas antes de su preparación, en el siguiente orden: longitud total, longitud de la cola vertebral, longitud de la pata trasera, longitud de la oreja y peso, determinando el sexo y su condición reproductora. En el caso de los murciélagos también se registró la longitud del antebrazo (Hall, 1962). Se recolectó un máximo de 3 ejemplares de cada especie autorizada (permiso especial de recolecta científica SGPA/
DGVS/7122 del 23 de septiembre de 2003) liberando en el mismo sitio los mamíferos que fueron fácilmente identificados y los considerados en alguna categoría de riesgo por la Norma Oficial Mexicana 059-SEMARNAT2001 (SEMARNAT, 2002). En todos los sitios de muestreo se realizó búsqueda de restos óseos, cadáveres y señales, así como observación directa de individuos vivos. Los ejemplares recolectados se preservaron siguiendo métodos convencionales (Hall, 1962) y fueron depositados en la Colección Mastozoológica (CMUM) de la Facultad de Biología de la Universidad Michoacana de San Nicolás de Hidalgo (FB-UMSNH). Para complementar los métodos de recolecta y observación, y revelar especies difíciles de detectar, se usó una cámara automática disparada por un detector infrarrojo (DeerCam®), colocada casi a nivel del suelo al lado de senderos y bebederos naturales en 10 sitios de muestreo (Chávez-León, 2005). La información de cada ejemplar registrado fue integrada a las bases de datos del modelo Biotica 4.1 de la Comisión Nacional para el Conocimiento y Uso de la Biodiversidad (CONABIO, 2003). Para la determinación taxonómica se usaron las claves de Hall (1981), Núñez y Pastrana (1990), Aranda (2000), Núñez (2002) y Apátiga y Núñez (2003). Una vez identificados los ejemplares, se procedió a elaborar el listado de especies conforme con la nomenclatura y secuencia taxonómica de Ceballos et al. (2005), que se basa en Wilson y Reeder (2005).

La distribución de cada especie se determinó usando los mapas de Hall (1981) y deVilla y Cervantes (2003), así como la información de Ceballos et al. (2002), RamírezPulido et al. (2005) y Ceballos et al. (2005). Su distribución fue clasificada como: endémica de México, endémica de México y Centroamérica, compartida con Norteamérica, compartida con Sudamérica, compartida con Norte y Sudamérica, y exótica. La afinidad zoogeográfica de los mamíferos registrados se clasificó como neártica, neotropical o transicional, considerando el esquema biogeográfico para México propuesto por Morrone (2005). Este esquema comprende 14 provincias biogeográficas agrupadas en 2 regiones, neártica y neotropical, y la zona de transición mexicana que incluye las provincias de la sierra Madre Oriental, sierra Madre Occidental, eje volcánico transmexicano, cuenca del Balsas y sierra Madre del Sur. En esta zona existe una mezcla de elementos neotropicales y neárticos que llegan a tal complejidad que no es posible asignarlos a la región neártica o a la neotropical (Escalante et al., 2005). El estado de conservación se basa en las categorías de riesgo de la NOM-059 (SEMARNAT, 2002), de la lista roja de la Unión Mundial para la Naturaleza (UICN, 2001) y en los apéndices de la CITES (UNEP, 2005).

Con los datos de las recolectas y observaciones se generó 
una curva de acumulación como función de la unidad de esfuerzo de recolecta y observación, usando como unidad los muestreos de 2 días (Chao, 2004; Escalante, 2004). Para estimar el número total esperado de especies $( \pm S)$ se usó el programa EstimateS 8 (Colwell 2006), empleando modelos no paramétricos y aleatorizando 200 veces con reemplazo. La riqueza de especies de mamíferos, o diversidad alfa, se determinó sumando las especies capturadas, observadas y fotografiadas.

\section{Resultados}

Durante el periodo de muestreo de campo (15 meses, 2003-2004) registramos una riqueza mastofaunística de 43 especies para el Parque Nacional Barranca del Cupatitzio. Estas especies pertenecen a 8 órdenes, 16 familias y 32 géneros (Cuadros 1 y 2).

El orden Carnivora presentó el mayor número de familias (5), seguido de Rodentia (3) y Chiroptera (3). Estos 2 últimos presentaron la mayor riqueza de especies (16 y 11 , respectivamente), constituyendo el $62.8 \%$ del total de la mastofauna del Parque Nacional Barranca del Cupatitzio, seguidos de Carnivora con $23.3 \%$. Didelphimorphia, Cingulata, Lagomorpha, Soricomorpha y Artiodactyla representan el $13.9 \%$ restante. Los mamíferos voladores constituyen el $25.6 \%$ del total de especies, mientras que los mamíferos no voladores comprenden el $74.4 \%$.

De las 43 especies de nuestro listado (Cuadro 2), $7(16.3 \%)$ son endémicas de México y $5(11.6 \%)$ de Mesoamérica. Las especies compartidas con Norteamérica son $10(23.3 \%)$, las compartidas con Sudamérica son 7 $(16.3 \%)$, las que tienen una distribución amplia que incluye Norte y Sudamérica son $13(30.2 \%)$ y 1 especie $(2.3 \%)$ es exótica con distribución mundial. Por sus afinidades biogeográficas, 22 especies $(52.4 \%)$ tienen afinidades neárticas, $16(38.1 \%)$ tienen afinidades neotropicales y $4(9.5 \%)$ son transicionales, principalmente endémicas de México. Hay 2 especies $(4.7 \%)$ del Parque Nacional Barranca del Cupatitzio consideradas en alguna categoría de riesgo en la Norma Oficial Mexicana 059 (SEMARNAT, 2002), de las que 1 se encuentra en peligro de extinción y 1 amenazada. A escala mundial, 1 mamífero de nuestra lista $(2.3 \%)$ se encuentra considerado como vulnerable en la clasificación de riesgos de extinción global de la UICN (2001). Una especie, se encuentra en el Apéndice I de CITES, que incluye especies amenazadas con extinción por el comercio internacional (UNEP, 2005).

Por último, se registró 1 especie exótica (Mus musculus) de distribución mundial, así como 2 domésticas que se han tornado ferales (Felis silvestris catus, Canis lupus domesticus; vid. Gentry et al., 2003). Su distribución en el parque es principalmente en edificaciones y zonas de mayor actividad humana, a excepción del perro feral, que también se encontró en las zonas más aisladas del Área de Montaña.

La curva de acumulación de especies observadas (Sobs) mostró un incremento creciente que no se estabilizó al término del muestreo (Fig. 2). Esta curva se generó con los datos de las especies recolectadas y observadas durante el trabajo de campo. Con base en los criterios definidos por Chazdon et al. (1998) para evaluar el desempeño de los diversos estimadores de la riqueza de especies se determinó que el estimador basado en la abundancia (ACE) fue el que mejor lo hizo, por lo que se eligió para el presente análisis. La curva generada mostró un rápido incremento que se estabilizó después de 18 muestreos (Fig. 2). El estimador ACE indica que pueden registrarse al menos $56 \pm 3.1$

Cuadro 1. Composición, riqueza de especies y estado de conservación de la mastofauna del Parque Nacional Barranca del Cupatitzio, Michoacán, México

\begin{tabular}{|c|c|c|c|c|c|}
\hline Órdenes & Familias & Géneros & $\begin{array}{c}\text { Especies } \\
(\%)\end{array}$ & $\begin{array}{c}\text { Especies } \\
\text { endémicas } \\
\text { (Michoacán, México, } \\
\text { Mesoamérica) }\end{array}$ & $\begin{array}{c}\text { Especies } \\
\text { en riesgo } \\
\text { (SEMARNAT, } \\
\text { IUCN, CITES) }\end{array}$ \\
\hline Didelphimorphia & 1 & 2 & $2(4.7)$ & 1 & 0 \\
\hline Cingulata & 1 & 1 & $1(2.3)$ & 0 & 0 \\
\hline Lagomorpha & 1 & 1 & $1(2.3)$ & 0 & 0 \\
\hline Soricomorpha & 1 & 1 & $1(2.3)$ & 1 & 0 \\
\hline Chiroptera & 3 & 7 & $11(25.6)$ & 2 & 0 \\
\hline Carnivora & 5 & 10 & $10(23.3)$ & 0 & 1 \\
\hline Artiodactyla & 1 & 1 & $1(2.3)$ & 0 & 0 \\
\hline Rodentia & 3 & 9 & $16(37.2)$ & 8 & 1 \\
\hline Total & 16 & 32 & 43 (100) & 12 & 2 \\
\hline
\end{tabular}


Cuadro 2. Mamíferos registrados en el Parque Nacional Barranca del Cupatitzio, Michoacán, México, 2003-2005. Se sigue el arreglo sistemático y la nomenclatura de Ceballos et al. (2005)

\begin{tabular}{lcccc}
\hline Medio de registro & Distribución & Afinidad & NOM- & UICN/ \\
zoogeográfica & 059 & Distribución en el & Parque \\
\hline
\end{tabular}

\section{DIDELPHIMORPHIA}

\section{Didelphidae}

Didelphis virginiana

Tlacuatzin canescens

CINGULATA

Dasypodidae

Dasypus novemcinctus

cad/hue

obs

$\mathrm{AM}$

$\mathrm{Na}$

Mt

Sylvilagus floridanus

SORICOMORPHA

Soricidae

Sorex saussurei

col

MA

Ztm

Mt

CHIROPTERA

Mormoopidae

Pteronotus parnellii

col

SA

$\mathrm{Nt}$

Mt

Phyllostomidae

Anoura geoffroyi

Artibeus hirsutus

Artibeus lituratus

Dermanura azteca

Dermanura phaeotis

Sturnira ludovici

\section{Vespertilionidae}

Myotis californicus

Myotis lucifugus

Myotis velifer

Lasiurus blossevillii

CARNIVORA

\section{Canidae}

Canis latrans

Urocyon cinereoargenteus

obs/hue/pel

obs/cad/fot

$\mathrm{AM}$

AM

$\mathrm{Na}$

Mt

Felidae

Leopardus wiedii

Lynx rufus

fot

obs/hue/exc

SA

NA

$\mathrm{Nt}$

$\mathrm{Na}$

Mt

Mt

\section{Mustelidae}

Mustela frenata

obs

AM

$\mathrm{Na}$

Mt-Ri

Mephitidae 
Cuadro 2. Continúa

\begin{tabular}{|c|c|c|c|c|c|c|}
\hline & Medio de registro & Distribución & $\begin{array}{c}\text { Afinidad } \\
\text { zoogeográica }\end{array}$ & $\begin{array}{l}\text { NOM- } \\
059\end{array}$ & $\begin{array}{l}\text { UICN/ } \\
\text { CITES }\end{array}$ & $\begin{array}{c}\text { Distribución en el } \\
\text { Parque }\end{array}$ \\
\hline Conepatus leuconotus & cad & NA & $\mathrm{Na}$ & & & Mt-Ri \\
\hline Mephitis macroura & cad & $\mathrm{AM}$ & $\mathrm{Na}$ & & & $\mathrm{Mt}$ \\
\hline Spilogale gracilis & cad & $\mathrm{AM}$ & $\mathrm{Na}$ & & & $\mathrm{Mt}$ \\
\hline \multicolumn{7}{|l|}{ Procyonidae } \\
\hline Nasua narica & obs/hue/fot & $\mathrm{AM}$ & $\mathrm{Nt}$ & & & Mt-Ri \\
\hline Procyon lotor & fot/cad/hue & $\mathrm{AM}$ & $\mathrm{Na}$ & & & Mt-Ri \\
\hline \multicolumn{7}{|l|}{ ARTIODACTYLA } \\
\hline Odocoileus virginianus & obs/hue & $\mathrm{AM}$ & $\mathrm{Na}$ & & & Mt \\
\hline \multicolumn{7}{|l|}{ RODENTIA } \\
\hline \multicolumn{7}{|l|}{ Sciuridae } \\
\hline Sciurus aureogaster & $\mathrm{col} / \mathrm{obs}$ & MA & $\mathrm{Na}$ & & & Mt-Ri \\
\hline Spermophilus variegatus & $\mathrm{col} / \mathrm{obs}$ & NA & $\mathrm{Na}$ & & & Mt-Ri \\
\hline \multicolumn{7}{|l|}{ Geomyidae } \\
\hline Cratogeomys fumosus & $\mathrm{col}$ & MX & Ztm & A & & $\mathrm{Mt}$ \\
\hline \multicolumn{7}{|l|}{ Muridae } \\
\hline Mus musculus & col & EX & & & & Mt-Ri \\
\hline Neotoma leucodon & col & NA & $\mathrm{Na}$ & & & Mt \\
\hline Neotoma mexicana & col & NA & $\mathrm{Na}$ & & & Mt \\
\hline Oryzomys couesi & col & MA & $\mathrm{Nt}$ & & & $\mathrm{Mt}$ \\
\hline Peromyscus gratus & col & NA & $\mathrm{Na}$ & & & $\mathrm{Mt}$ \\
\hline Peromyscus spicilegus & col & MX & Ztm & & & Mt \\
\hline Reithrodontomys chrysopsis & col & MX & Ztm & & & Mt \\
\hline Reithrodontomys fulvescens & col & NA & $\mathrm{Na}$ & & & $\mathrm{Mt}$ \\
\hline Reithrodontomys megalotis & col & NA & $\mathrm{Na}$ & & & Mt \\
\hline Reithrodontomys mexicanus & col & SA & $\mathrm{Nt}$ & & & Mt \\
\hline Reithrodontomys sumischrasti & col & MA & $\mathrm{Nt}$ & & & Mt \\
\hline Sigmodon alleni & col & MX & $\mathrm{Nt}$ & & & Mt \\
\hline Sigmodon mascotensis & col & MX & $\mathrm{Nt}$ & & & Mt \\
\hline
\end{tabular}

Medio de registro: obs $=$ observado, $\mathrm{col}=$ colectado, cad $=$ cadáver con cráneo, hue $=$ huellas, pel $=$ pelo, exc $=$ excretas, fot $=$ foto trampa. Distribución: MX = endémica de México, MA = endémica de Mesoamérica, NA= compartida con Norteamérica, $\mathrm{SA}=$ compartida con Sudamérica, $\mathrm{AM}=$ compartida con Norte y Sudamérica, EX = exótica. Afinidad zoogeográfica: $\mathrm{Na}=$ neártica, $\mathrm{Nt}$ = neotropical, $\mathrm{Ztm}=$ zona de transición mexicana. NOM-059 (categorías de riesgo conforme a la NOM-059-SEMARNAT-2001): $\mathrm{P}$ = peligro de extinción, A = amenazada. UICN/CITES (categorías mundiales de riesgo de extinción): $\mathrm{VU}=$ vulnerable $(\mathrm{UICN})$, I = Apéndice I (CITES). Distr. en el Parque (distribución en el Parque Nacional Barranca del Cupatitzio): Mt = área de montaña, Ri = área de río.

especies, lo que sugiere la posible presencia de 13 más que las registradas en nuestro trabajo de campo, por lo que la riqueza obtenida equivale al $77 \%$ de las potenciales en la zona de estudio.

\section{Discusión}

Nuestros datos representan un incremento del 25.6 $\%$ en la riqueza de mamíferos del Parque Nacional en comparación con las 32 especies registradas por Orduña 


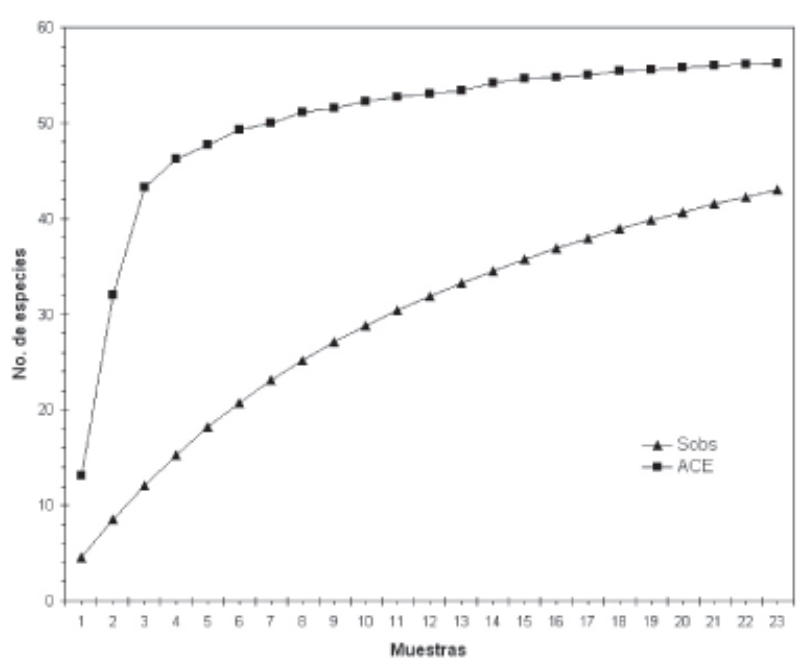

Figura 2. Curvas de acumulación de especies observadas (Sobs) y estimador no paramétrico de riqueza de especies de mamíferos (ACE) en el Parque Nacional Barranca del Cupatitzio, Michoacán, México.

et al. (1993), que realizaron su trabajo de campo en 1984 y 1985 pero no indican su esfuerzo de muestreo. El total de especies registradas en el parque equivale al $69.3 \%$ de los mamíferos de la región de la sierra Purépecha (Orduña y Salas, 1993), al 26.9\% de los mamíferos de Michoacán (Núñez, 2005) y el $9.4 \%$ de los mamíferos continentales de México (Ceballos et al., 2005). Se puede considerar que este Parque Nacional alberga una alta riqueza de especies tomando en cuenta que ocupa una superficie muy pequeña $(0.00024 \%$ de la superficie nacional y el $0.0079 \%$ de la superficie estatal).

Torres et al. (2003) registraron 25 especies de mamíferos no voladores, pertenecientes a 12 familias y 7 órdenes en 4189 ha de bosques de coníferas en la vecina Comunidad Indígena de Nuevo San Juan Parangaricutiro, mientras que Zaragoza (2006) registró para el mismo grupo 34 especies de 13 familias y 7 órdenes en 458 ha. Aunque la cantidad de órdenes y familias es muy similar, en la comunidad indígena hay una menor riqueza de especies, lo que probablemente se debe a los efectos de la alteración y fragmentación causada en sus bosques, por un lado, por los aprovechamientos forestales, la resinación de pinos, la fruticultura y la ganadería extensiva que se practican ahí (Torres et al., 2003), y por otro, de eventos de tipo natural como es la erupción reciente del volcán Paricutín (Burt, 1961). Aunque existe poca diversidad de tipos de vegetación en el parque, la mayor riqueza de especies puede deberse a su heterogeneidad topográfica y la alta diversidad estructural de la vegetación como consecuencia de su protección y la exclusión por más de
70 años de actividades humanas, como las practicadas en los bosques Nuevo San Juan y en la sierra Purépecha en general. Es posible que la discrepancia en el número de especies registradas en la zona respecto a lo obtenido en este trabajo sea el resultado de un esfuerzo de muestreo considerablemente mayor.

Al estar este Parque Nacional fragmentado en 2 secciones separadas entre sí por un espacio ocupado por asentamientos humanos, existen diferencias en riqueza de especies entre ambas áreas: en el Área de Montaña se registraron 31 especies exclusivas, mientras que en el área de río no se registraron especies exclusivas, solamente 12 especies compartidas con la otra zona. La diferencia de riqueza de mamíferos entre las 2 áreas del parque indica la existencia de un bajo intercambio de especies y revela la inexistente conectividad entre ambas (McGarigal y Marks, 1995). Esto se debe a que el área de río, con el $27.9 \%$ de las especies registradas, es un fragmento de vegetación alterada, de pequeña extensión, que se encuentra aislado dentro de la zona urbana de la ciudad, donde hay una constante presencia humana, visitada por más de 750000 paseantes al año, y donde se registró la mayor presencia de perros y gatos ferales, al igual que ratones domésticos. En cambio, el área de montaña, con el $100 \%$ del total de especies, tiene una extensión 22 veces mayor, donde la vegetación se mantiene en buen estado de conservación y está en conexión con las zonas boscosas al norte y poniente, aunado a poca actividad humana. Lo anterior sugiere que esta área aún mantiene un buen nivel de integridad biológica, a diferencia del área de río que puede haber sufrido un proceso de defaunación por su aislamiento y degradación (Cafaro y Primack, 2001).

En este trabajo no fueron detectadas 9 especies que registraron Orduña et al. (1993) hace más de 20 años para el área de estudio (Macrotus waterhousii, Desmodus rotundus, Anoura geoffroyi, Hylonicterus underwoodi, Leptonycteris nivalis, Myotis thysanodes, Bassariscus astutus y Zygogeomys trichopus), por lo que posiblemente ya no existen en el lugar. Zaragoza (2006) registra 2 tuzas (Cratogeomys tylorhinus y C. gymnurus), pero aquí se sigue la recomendación de Ceballos et al. (2005), fundamentada en estudios moleculares, de agruparlas con Cratogeomys fumosus. Igualmente, se sigue la recomendación de esos autores en cuanto a que Sigmodon hispidus, registrada por Zaragoza (2006), ya no se encuentra en México. Aquí se reporta $S$. alleni, ya que estudios de secuencias de nucleótidos indican que ésta y $S$. hirsutus son especies hermanas.

El endemismo observado en este lugar $(16.3 \%)$ es menor que el registrado para México (135 de 457 mamíferos continentales, 29.5\%; Ceballos et al., 2005). Entre las especies endémicas registradas destaca la presencia de 
Tlacuatzin canescens, Artibeus hirsutus, Cratogeomys fumosus, Peromyscus spicilegus y Reithrodontomys chrisopsis. Esta última es endémica del eje volcánico transmexicano (Villa y Cervantes, 2003).

El parque se ubica en el área de transición de la zona templada del eje neovolcánico transmexicano y la tropical seca de la depresión del Balsas, provincias bióticas que son consideradas como importantes centros de endemismo de mamíferos (Fa y Morales, 1993; Escalante et al., 2005). Nuestros resultados confirman que la mastofauna de esta zona es una mezcla de elementos tanto de origen neártico como neotropical e incluye especies transicionales, endémicas del centro de México.

De las 229 especies de mamíferos que están consideradas por la NOM-059-SEMARNAT-2001 como en peligro, amenazadas o bajo protección especial (SEMARNAT, 2002), únicamente 2 (0.9\%; Leopardus wiedii y $C$. fumosus) se encuentran en Barranca del Cupatitzio, registradas en una ocasión, las que representan el $4.7 \%$ del total de especies del parque. En el caso de $L$. wiedii se trata de un registro en el limite de su área de distribución que originalmente se identificó como L. pardalis (ChávezLeón, 2005). Sin embargo, una comparación reciente con fotos de ejemplares de estas 2 especies tomadas en Chamela, Jalisco, también con cámaras automáticas, indican que, por diferencias en el grosor, las manchas y la proporción de la longitud de la cola respecto al cuerpo, así como la cabeza con grandes ojos y orejas, se trata en realidad de L. wiedii. En el caso de C. fumosus es una especie poco abundante debido a la baja disponibilidad de hábitat adecuado, ya que la mayor parte del parque está cubierto por vegetación boscosa con dosel cerrado que en pocas zonas está en contacto con áreas de cultivo o abiertas
(Cervantes et al., 1993).

Un resultado importante de este inventario es la identificación de especies prioritarias para su conservación y manejo en el parque. Una jerarquización adecuada debe considerar a las especies catalogadas en alguna categoría de riesgo, a las endémicas y a las de distribución restringida. En este sentido, las especies se pueden jerarquizar de mayor a menor prioridad considerando criterios de riesgo, endemismo y área de distribución (Bibby et al., 1992; Ceballos et al., 1998). Aplicando estos conceptos a los mamíferos del parque, las especies con mayor prioridad incluyen aquéllas consideradas como en peligro de extinción o amenazadas y que son endémicas de distribución restringida (A), seguidas de las que están en esas categorías de riesgo pero son de amplia distribución (B), y de las endémicas de distribución restringida pero no consideradas en riesgo (C). Conforme a estos criterios, en el parque se encuentran 8 especies prioritarias para su conservación (Cuadro 3), por lo que en el Programa de Conservación y Manejo se establecieron actividades para determinar su estacionalidad, distribución y tamaño poblacional, además de implantar un sistema de monitoreo y designar áreas de protección dentro del parque. El monitoreo permitirá determinar el efecto de las actividades generales de conservación y manejo propuestas en el PCyM sobre las fluctuaciones de las poblaciones de las especies prioritarias.

La pérdida de los bosques de coníferas del eje neovolcánico por tala clandestina, expansión de la agricultura y en particular en la zona de Uruapan por el cultivo del aguacate y la expansión urbana desordenada, hacen del Parque Nacional Barranca del Cupatitzio un área importante para la conservación de la diversidad

Cuadro 3. Especies prioritarias del Parque Nacional Barranca del Cupatitzio, Michoacán, México

\begin{tabular}{|c|c|c|c|c|c|}
\hline Especie & Prioridad & NOM-059-2001 & $I U C N$ & CITES & Distribución \\
\hline Cratogeomys fumosus & A & A & & & MX \\
\hline Leopardus wiedii & B & $\mathrm{P}$ & & I & SA \\
\hline Artibeus hirsutus & B & & VU & & MX \\
\hline Peromyscus spicilegus & $\mathrm{C}$ & & & & MX \\
\hline Reithrodontomys chrysopsis & $\mathrm{C}$ & & & & MX \\
\hline Sigmodon alleni & $\mathrm{C}$ & & & & MX \\
\hline Sigmodon mascotensis & $\mathrm{C}$ & & & & MX \\
\hline Talcuatzin canescens & $\mathrm{C}$ & & & & MX \\
\hline
\end{tabular}

Prioridad: $\mathrm{A}$ = en alguna categoría de riesgo y endémica de distribución restringida, $\mathrm{B}=$ en alguna categoría de riesgo pero de amplia distribución, $\mathrm{C}$ = endémica de distribución restringida pero no considerada en riesgo. NOM-059-SEMARNAT2001: P = peligro de extinción, A = amenazada. IUCN (Unión Internacional para la Conservación de la Naturaleza): VU = vulnerable. CITES: I = Apéndice I (en peligro de extinción por comercio ilegal). Distribución: MX = endémica de México, $\mathrm{SA}=$ distribuida de México a Sudamérica. 
de mamíferos del eje volcánico transmexicano. Por su cercanía con la zona forestal de la comunidad indígena de Nuevo Parangaricutiro y el Parque Nacional Pico de Tancítaro puede ser considerado como una extensión de ese corredor biológico.

\section{Agradecimientos}

Al Consejo Nacional de Ciencia y Tecnología (CONACYT-SEMARNAT 2002-C01-0196/A-1), a la Comisión Nacional para el Conocimiento y Uso de la Biodiversidad (CONABIOFB919/AS014/03) y al Patronato del Parque Nacional Barranca del Cupatitzio, A. C. por su apoyo financiero para el proyecto "Inventario Florístico y Faunístico del Parque Nacional Barranca del Cupatitzio, Michoacán”. A Arturo Núñez Garduño por su apoyo como director de tesis de SZR. A Guillermo Sánchez López por permitirnos usar sus datos de los murciélagos del parque. A Julio Valdés, Luis Alcauter, Javier Salvador Almanza, Neftalí Mendoza, Jaime Boyzo, Claudia Zamora, Nancy Silva, Ana L. Báez, Alejandra Huerta y Montserrat Orozco por su ayuda en el trabajo de campo. Rodrigo Núñez Pérez proporcionó fotos de tigrillo y ocelote de Chamela y señaló características que permitieron reconocer las diferencias entre estas 2 especies e identificar correctamente al ejemplar de Leopardus wiedii aquí registrado. Dos revisores anónimos contribuyeron a mejorar este escrito.

\section{Literatura citada}

Aranda, M. 2000. Huellas y otros rastros de los mamíferos grandes y medianos de México. Instituto de Ecología, Xalapa, Veracruz. 212 p.

Apátiga C., M. C. y A Núñez G. 2003. Claves para la identificación taxonómica de los mamíferos michoacanos. Coordinación de la Investigación Científica, Universidad Michoacana de San Nicolás de Hidalgo, Morelia, Michoacán. 73 p.

Bello G., M.A. y X. Madrigal S. 1996. Estudio florístico del Campo Experimental "Barranca del Cupatitzio", Uruapan, Michoacán. Folleto Científico 2, Campo Experimental Uruapan, Instituto Nacional de Investigaciones Forestales, Agrícolas y Pecuarias.

Bibby, C. J., N. J. Collar, M. J. Crosby, M. J. Gead, Ch. Imboden, T. H. Johnson, A. J. Stattersfield y S. J. Thirgood. 1992. Putting biodiversity on the map: priority areas for global conservation. ICBP International Council for Bird Preservation, Cambridge. 90 p.

Burt, H. W. 1961. Some effects of volcán Paricutín on vertebrates. University of Michigan, Occasional Papers Museum of Zoology 620:1-24.

Cafaro, P. y R. Primack. 2001. Ecological integrity: evaluating success in national parks and protected areas. In Encyclopedia of life sciences. Wiley, Chichester. p. 1-6.

Ceballos, G., P. Rodríguez y R. A. Medellín. 1998. Assessing conservation priorities in megadiverse Mexico: mammalian diversity, endemicity, and endangerment. Ecological Applications 8:8-17.

Ceballos, G., J. Arroyo-Cabrales y R. A. Medellín. 2002. The mammals of Mexico: composition, distribution, and conservation status. Ocassional Papers, Museum of Texas Tech University 218:1-27.

Ceballos, G., J. Arroyo-Cabrales, R. A. Medellín y Y. DomínguezCastellanos. 2005. Lista actualizada de los mamíferos de México. Revista Mexicana de Mastozoología 9:27-71.

Cervantes, F. A., V. J. Sosa, J. Martínez, R. M. González y R. C. Dowler. 1993. Pappogeomys tylorhinus. Mammalian Species 443:1-4.

Chao, A. 2004. Species richness estimation. In N. Balakrishnan, C. B. Read y. Vidakovic (eds.) Encyclopedia of Statistical Sciences. Wiley, New York. p. 1-23

Chávez-León, G. 2005. A recent record of Lepardus pardalis (Linnaeus, 1758) from Michoacán, Mexico. Revista Mexicana de Mastozoología 9:122-126.

Chazdon, R. L., R. K. Colwell, J. S. Denslow y M. R. Guariguata. 1998. Statistical methods for estimating species richness of woody regeneration in primary and secondary rain forests of NE Costa Rica. In F. Dallmeier y J. A. Comiskey (eds.). Forest biodiversity research, monitoring and modeling: conceptual background and Old World case studies. Man and the Biosphere Series, vol. 20, UNESCO and Parthenon, Paris. p. 285-309.

Colwell, R. K. 2006. EstimateS: statistical estimation of species richness and shared species from samples. Version 8. User's guide and application. <http/purl.oclc.org/estimates> Consultado: 19 septiembre 2008.

CONABIO (Comisión Nacional para el Conocimiento y Uso de la Biodiversidad). 2003. Sistema de información biótica. Versión 4.1. Manual de usuario. Fideicomiso Fondo para la Biodiversidad, CONABIO, México, D. F. 704 p.

Escalante E., T. 2004. ¿Cuántas especies hay? Los estimadores no paramétricos de Chao. Revista Elementos 52:53-56.

Escalante, T., G. Rodríguez y J. J. Morrone. 2005. Las provincias biogeográficas del componente mexicano de montaña desde la perspectiva de los mamíferos continentales. Revista Mexicana de Biodiversidad 76:199-205.

Fa, J. E. y L. M. Morales. 1993. Patterns of mammalian diversity in Mexico. In Biological diversity of Mexico: origins and distribution, T. P. Ramamoorthy, R. Bye, A. Lot y J. Fa (eds.) Oxford University Press, New York. p. 281-299.

García, E. 1981. Modificaciones al sistema de clasificación climática de Köppen. Larios, México, D. F. 252 p.

Gentry, A., J. Clutton-Brock y C. P. Groves. 2003. Opinion 2027. Usage of 17 specific names based on wild species which are pre-dated by or contemporary with those based on domestic animals (Lepidoptera, Osteichthyes, Mammalia): conserved. Bulletin of Zoological Nomenclature 60:81-84.

Gómez-Tagle R., A. F. 1985. Levantamiento de suelos del Campo Experimental Forestal Barranca del Cupatitzio, y sus relaciones con la vegetación de coníferas. Tesis, Maestría Facultad de Ciencias, UNAM, México, D.F. 
Hall, E. R. 1962. Collecting and preparing study specimens of vertebrates. Miscellaneous Publication, Museum of Natural History, University of Kansas 30:1-46.

Hall, E. R. 1981. The mammals of North America, segunda edición, vols. I y II. Wiley, New York. 1181 p.

INEGI (Instituto Nacional de Estadística, Geografía e Informática). 2006. II Conteo Nacional de Población y Vivienda 2005. Aguascalientes, Aguascalientes.

Janzen, D. H. 1997. Wildland biodiversity management in the tropics. In Biodiversity. II. Understanding and protecting our biological resources, M. L. Reaka-Kudla, D. E. Wilson y E. O. Wilson (eds.) Joseph Henry, Washington, D. C. p. 411-411.

McGarigal, K. y B. J. Marks. 1995. Fragstats: spatial pattern analysis program for quantifying landscape structure. General Technical Report PNW-GTR-351. USDA-Forest Service, Portland, Oregon.

Morrone, J. J. 2005. Hacia una síntesis biogeográfica de México. Revista Mexicana de Biodiversidad 76:207-252.

Núñez G., A. 2002. Los mamíferos del orden Carnivora en Michoacán. Facultad de Biología, Universidad Michoacana de San Nicolás de Hidalgo, Morelia, Michoacán, México. $105 \mathrm{p}$.

Núñez G., A. 2003. Mamíferos silvestres del estado de Michoacán. Lista actualizada, distribución y estatus de meta poblaciones. Biológicas 5:30-39.

Núñez G., A. 2005. Mamíferos. In La biodiversidad de Michoacán. Estudio de estado, L. E. Villaseñor G. (ed.) Comisión Nacional para el Conocimiento y Uso de la Biodiversidad/ Secretaría de Urbanismo y Medio Ambiente/Universidad Michoacana de San Nicolás de Hidalgo, Morelia, Michoacán. p. 104-105.

Núñez G., A. y G. Pastrana H. 1990. Los roedores michoacanos. Manual de identificación. Coordinación de la Investigación Científica, UMSNH, Morelia, Michoacán. 124 p.

Orduña T., C. y M. A. Salas P. 1993. Los mamíferos de la sierra Purépecha del estado de Michoacán. Boletín Divulgativo 78, Instituto Nacional de Investigaciones Forestales y Agropecuarias, México, D. F. 32 p.

Orduña T., C., M. A. Salas P y P. Gaytán T. 1993. Aves y mamíferos del Campo Experimental Forestal "Barranca del Cupatitzio", Uruapan, Michoacán. Folleto Técnico 7, Centro de Investigaciones del Pacífico Centro, Instituto Nacional de Investigaciones Forestales y Agropecuarias, Guadalajara, Jalisco. $16 \mathrm{p}$.

Ramírez-Pulido, J., J. Arroyo-Cabrales y A. Castro-Campillo.
2005. Estado actual y relación nomenclatural de los mamíferos terrestres de México. Acta Zoológica Mexicana (n. s.) 21:21-82.

Romero-Almaraz, M. L., C. Sánchez-Hernández, C. GarcíaEstrada y R. D. Owen. 2000. Mamíferos pequeños. Manual de técnicas de captura, preparación, preservación y estudio. Universidad Nacional Autónoma de México y Universidad Autónoma del Estado de México, México, D. F. 151 p.

Rzedowski, J. 1978. Vegetación de México. Limusa, México, D. F. 432 p.

SEMARNAT (Secretaría de Medio Ambiente y Recursos Naturales). 2002. Norma Oficial Mexicana NOM-059ECOL-2001, Protección ambiental-Especies nativas de México de flora y fauna silvestres-Categorías de riesgo y especificaciones para su inclusión, exclusión o cambioLista de especies en riesgo. Diario Oficial de la Federación, segunda sección, 6 de marzo de 2002, p. 95-190.

Torres, A., A. Velázquez y J. Lobato. 2003. Riqueza, diversidad y patrones de distribución espacial de los mamíferos. In Las enseñanzas de San Juan. Investigación participativa para el manejo integral de recursos naturales. A. Velázquez, A. Torres y G. Bocco (comp.) Instituto Nacional de Ecología, Secretaría de Medio Ambiente y Recursos Naturales, México, D. F. p. 277-299.

UICN (Unión Internacional para la Conservación de la Naturaleza). 2001. Categorías y criterios de la Lista roja de la UICN: versión 3.1. Comisión de Supervivencia de Especies de la UICN. IUCN, Gland . 33 p.

UNEP. 2005. CITES handbook manual guide. United Nations Environment Programme, Gland. 438 p.

Vargas M., F. 1997. Parques nacionales de México. Aspectos físicos, sociales, legales, administrativos, recreativos, biológicos, culturales, situación actual y propuestas en torno a los parques nacionales de México. Instituto Nacional de Ecología, México, D. F. 343 p.

Villa R., B. y F. A. Cervantes. 2003. Los mamíferos de México. Iberoamérica, México, D. F. 140 p.

Wilson, D. E. y D. M. Reeder (eds.). 2005. Mammal species of the world. A taxonomic and geographic reference, tercera edición. Johns Hopkins University Press, Baltimore, Maryland.

Zaragoza R., S. E. 2006. Mamíferos no voladores del Parque Nacional "Barranca del Cupatitzio" Uruapan, Michoacán, México. Tesis, Facultad de Biología, Universidad Michoacana de San Nicolás de Hidalgo, Morelia, Michoacán. 155 p. 